\title{
DEFORMATION MECHANISMS OPERATING DURING PLASTIC FLOW OF AN AZ63 MAGNESIUM ALLOY STUDIED BY THE STRESS RELAXATION TECHNIQUE
}

\begin{abstract}
Magnesium alloy AZ63 (Mg-6Al-3Zn) was deformed at temperatures between room temperature and $300{ }^{\circ} \mathrm{C}$. Stress relaxation tests were performed in order to reveal features of the thermally activated dislocation mechanism. Internal and effective components of the applied stress were estimated. Very high values of the internal stress estimated at lower temperatures decrease rapidly with increasing deformation temperature. The apparent activation volume decreases with increasing effective stress. The values of the activation volume as well as the activation enthalpy indicate that the main thermally activated process is most probably the glide of dislocations in non-compact planes. Nonmonotonous temperature dependence of the flow stress was observed. Post relaxation effect was detected. Strain ageing effects are explained by the dynamic solute-dislocation interaction.
\end{abstract}

Key words: Magnesium alloy; Stress relaxation; Thermally activated dislocation motion; Dynamic strain ageing

\section{Introduction}

Most used commercial magnesium alloys of an AZ series exhibit a relatively high specific strength (the strength/density ratio) at room temperature but poor mechanical properties at elevated temperatures [1-4]. These alloys exhibit universally the hexagonal close packed structure with the limited number of slip systems in the basal plane. It strongly influences the ductility of such alloys at lower temperatures. The ductility increases at elevated temperatures due to possibility of the activation of non-basal slip systems.

During plastic deformation in a certain range of temperature and strain rate, different micro-mechanisms may play an important role. The analysis of deformation microstructures has shown that one should consider dislocation-based mechanisms in order to explain the deformation behaviour. It is widely accepted that the resolved shear stress, $\tau$, and necessary for dislocation motion in the slip plane can be divided into two components:

$$
\tau=\tau_{i}+\tau^{*}
$$

where $\tau_{i}$ is the (internal) athermal contribution to the stress, resulting from long-range internal stresses impeding the plastic flow and the effective shear stress, $\tau^{*}$, acts on dislocations during their thermally activated dislocation motion. The mean velocity of dislocations, $v$, is connected with the plastic shear strain rate by the Orowan equation:

$$
\dot{\gamma}=\rho_{m} b v .
$$

\footnotetext{
* Z. Trojanova, P. Lukac

Department of Physics of Materials, Faculty Mathematics and Physics, Charles University, Prague, Czech Republic,

E-mail: ztrojan@met.mff.cuni.cz
}

In polycrystalline materials, the resolved shear stress, $\tau$, and its components are related to the applied stress, $\sigma$, and its corresponding components by the Taylor orientation factor $\psi$ : $\sigma=\psi \tau$. A simple relation between the resolved shear strain rate and strain rate is $\gamma=\psi \varepsilon$, then the flow stress

$$
\sigma=\sigma_{i}+\sigma^{*}
$$

The internal stress component $\sigma_{i}$ is done as

$$
\sigma_{i}=\alpha G b \rho_{t}^{1 / 2}
$$

where $G$ is the shear modulus, $\alpha$ is a constant describing interaction between dislocations, $b$ is the Burgers vector of dislocations, and $\rho_{t}$ is the total dislocation density. The effective stress component, $\sigma^{*}$, is defined by the Arrhenius equation (5). The plastic strain rate, $\dot{\varepsilon}$, for a single thermally activated process can be expressed as:

$$
\dot{\varepsilon}=\dot{\varepsilon}_{0} \exp \left[-\frac{\Delta G\left(\sigma^{*}\right)}{k T}\right],
$$

where $\dot{\varepsilon}_{0}$ is a pre-exponential factor containing the mobile dislocation density, the average area covered by the dislocations in each activation process, the dislocation Burgers vector, the vibration frequency of the dislocation line, and the geometric factor. $T$ is the absolute temperature and $k$ is the Boltzmann constant. $\Delta \mathrm{G}\left(\sigma^{*}\right)$ is the change in the Gibbs free enthalpy depending on the effective stress $\sigma^{*}=\sigma-\sigma_{i}$ and its simple form is

$$
\Delta G\left(\sigma^{*}\right)=\Delta G_{0}-V \sigma^{*}=\Delta G_{0}-V\left(\sigma-\sigma_{i}\right) .
$$


Here $\Delta G_{0}$ is the Gibbs free enthalpy necessary for overcoming a short-range obstacle without stress and $V=b d L$ is the activation volume where $d$ is the obstacle width and $L$ is the mean length of dislocation segments between obstacles. It should be mentioned that $L$ may depend on the stress acting on dislocation segments.

The aim of the present work is to investigate deformation behaviour of $\mathrm{Mg}-6 \mathrm{Al}-1 \mathrm{Zn}$ alloy at different temperatures and to reveal the mechanisms responsible for plastic deformation of the alloy at elevated temperatures.

\section{Experimental procedure}

Magnesium alloy AZ63 (the nominal composition Mg-6Al$1 \mathrm{Zn}$ ), in wt. \%, was gravity cast. Samples were deformed in compression in an INSTRON 5882 machine at a constant cross head-speed giving an initial strain rate of $2.8 \times 10^{-5} \mathrm{~s}^{-1}$ over a temperature range of 23 to $300{ }^{\circ} \mathrm{C}$. Sequential stress relaxation (SR) tests were performed at increasing stress along a stress-strain curve. Duration of the SR was $300 \mathrm{~s}$. Components of the applied stress $\left(\sigma_{i}, \sigma^{*}\right)$ were estimated using Li's method $[5,6]$. The SR curves were fitted to a power law function in the form:

$$
\sigma-\sigma_{i}=[a(m-1)]^{\frac{1}{1-m}}\left(t+t_{0}\right)^{\frac{1}{1-m}},
$$

where $a, t_{0}$, and $m$ are fitting parameters.

The stress relaxation technique has been demonstrated to be a useful experimental method for estimating the activation volume and hence for determining thermally activated process(es). In a stress relaxation test, the specimen is deformed to a certain stress $\sigma_{0}$ and then the machine is stopped and the stress is allowed to relax. The stress decreases with time $t$. The specimen can be again reloaded and deformed to a higher stress and the stress relaxation test may be repeated. The time derivative $\dot{\sigma}=\mathrm{d} \sigma / \mathrm{d} t$ is the stress relaxation rate and $\sigma=\sigma(t)$ is the flow stress at time $t$ during the SR. Stress relaxation tests are very often analysed under the assumption that the stress relaxation rate is proportional to the strain rate $\dot{\varepsilon}$ according to [7]:

$$
\dot{\varepsilon}=-\dot{\sigma} / M
$$

where $M$ is the combined modulus of the specimen machine set.

$$
\begin{aligned}
& \text { Combining (5), (6) and (8), we have } \\
& -\dot{\sigma}=M \dot{\varepsilon}_{0} \exp \left[-\frac{\Delta G_{0}-V \sigma^{*}}{k T}\right] .
\end{aligned}
$$

Taking the logarithm of this equation we get

$$
\ln (-\dot{\sigma})=\ln \left(M \dot{\varepsilon}_{0}\right)-\frac{\Delta G_{0}}{k T}+\frac{V \sigma^{*}}{k T} .
$$

The stress decrease with time during the SR can be described by the well known Feltham equation [8]:

$$
\Delta \sigma(t)=\sigma(0)-\sigma(t)=\alpha \ln (\beta t+1),
$$

where $\sigma(0) \equiv \sigma_{0}$ is the stress at the beginning of the stress relaxation at time $t=0$,

$$
\begin{aligned}
& \alpha=\frac{k T}{V}, \\
& \beta=\frac{M \dot{\varepsilon}_{0} V}{k T} \exp \left[-\frac{\Delta G_{0}-V \sigma^{*}(0)}{k T}\right]=\frac{M \dot{\varepsilon}(0)}{\alpha},
\end{aligned}
$$

where $\dot{\varepsilon}(0)$ is the plastic strain rate at the beginning of the relaxation.

\section{Results and discussion}

\subsection{Internal stress}

Parts of the true stress-true strain curves measured at various temperatures are introduced in Fig. 1. Full points (depicted as $\left.\sigma_{a p}\right)$ indicate the stresses at which the SR tests were performed. Figs 1a-1e demonstrate the development of the internal stress $\sigma_{i}$ with temperature. While the internal stress is at ambient temperature and $50{ }^{\circ} \mathrm{C}$ a substantial contribution to the applied stress $\sigma_{a p}$. The effective stress component $\sigma^{*}$ forms at lower temperature small contribution to the applied stress (Fig. 1a.b) but it increases with increasing temperature and at $300{ }^{\circ} \mathrm{C}$ is higher then the internal stress (Fig. 1e). The internal stress depends on the dislocation density, i.e. $\sigma_{i} \propto \rho^{1 / 2}$. A decrease of the internal stress indicates a decrease of the dislocation density. In the case when the internal stress is approximately constant or slightly decreasing with strain (see Fig. 1c), some equilibrium between multiplication and annihilation of dislocations may be considered. The decrease of the dislocation density with temperature is also visible from Fig. 2, where the temperature dependences of the ratio $\sigma_{i} / \sigma_{a p}$ are shown. It should be noted that the values were estimated from SR test performed at $\varepsilon \sim 0.01$. The internal stress of several magnesium alloys was studied by Milicka et al. [9] in creep experiments. They

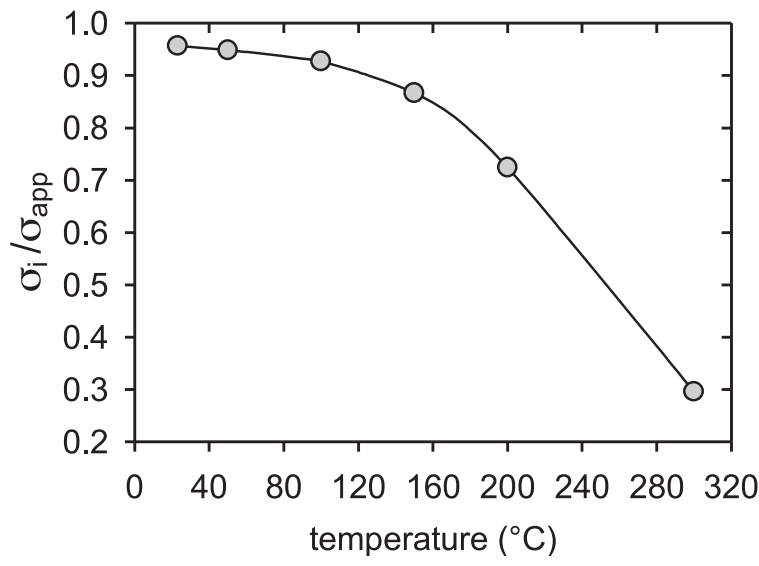

Fig. 2. Variation of the internal/applied stress ratio (obtained at the strain of about 1\%) with temperature. 


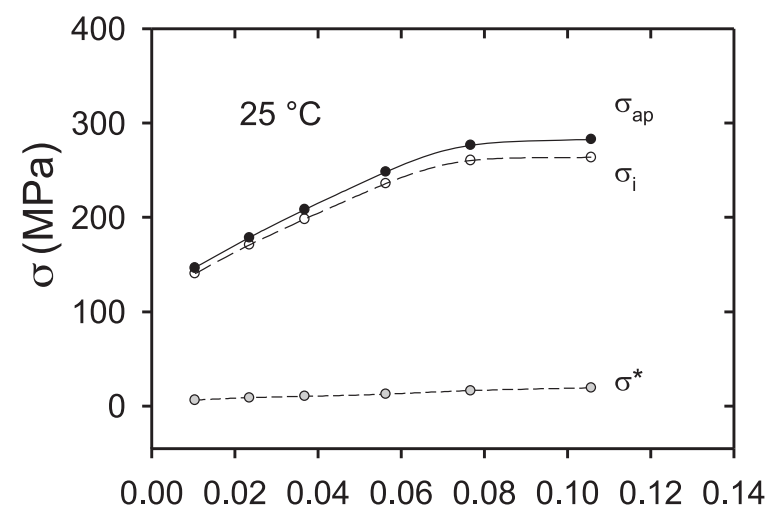

a)

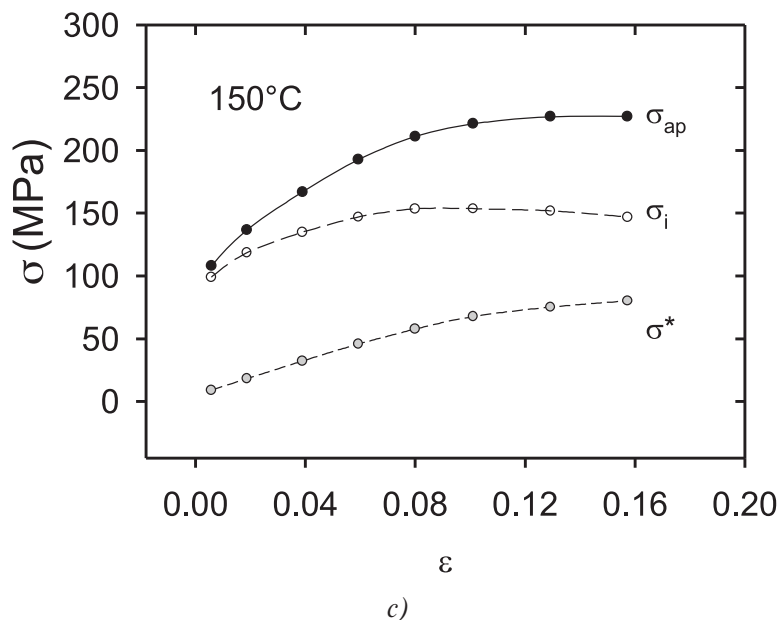

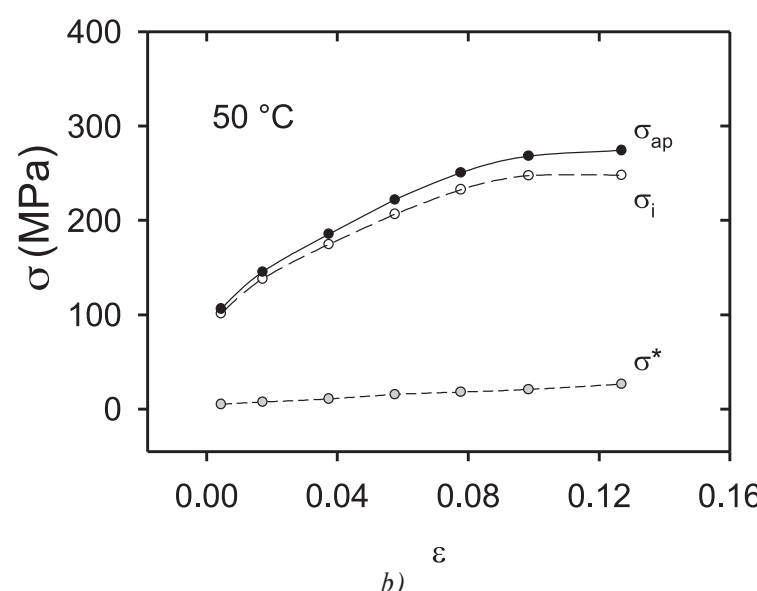

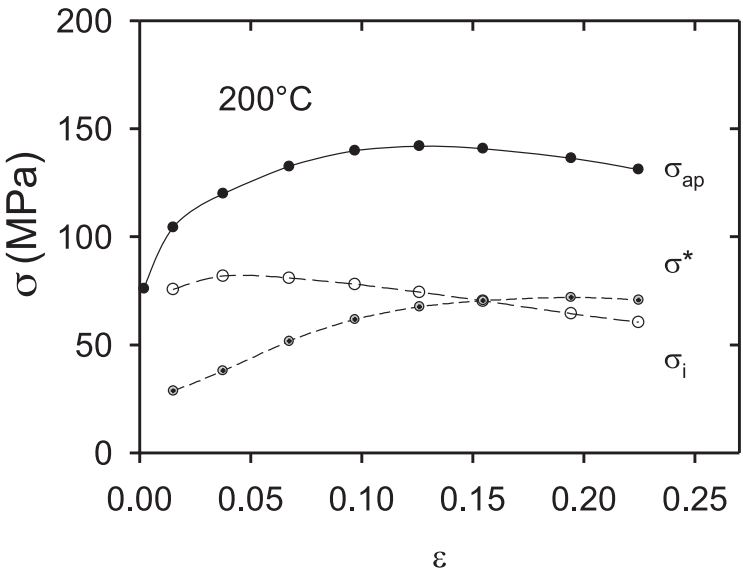

d)

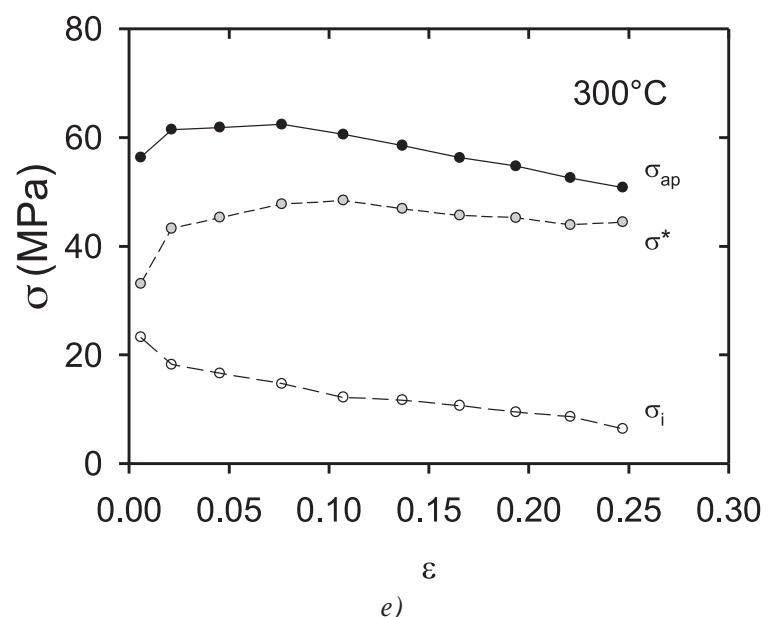

Fig. 1. Parts of the true stress-true strain curves obtained at various temperatures. The points of oap on the curves indicate the stresses at which the SR tests were performed: a) $25^{\circ} \mathrm{C}$, b) $\left.50{ }^{\circ} \mathrm{C}, \mathrm{c}\right) 150{ }^{\circ} \mathrm{C}$, d) $200{ }^{\circ} \mathrm{C}$, e) $300{ }^{\circ} \mathrm{C}$. 


\subsection{Thermal activation}

The activation volume was estimated using Eq. (11). The values of the activation volume (as usual divided by $b^{3}$ ) estimated at four temperatures are introduced in Fig. 3. Four discrete decreasing curves were observed.

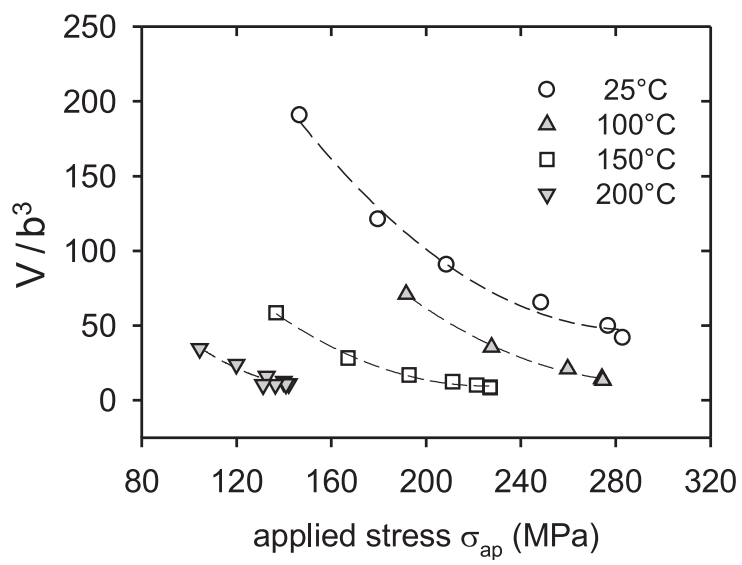

Fig. 3. Plot of the apparent activation volume (in $b^{3}$ ) against the applied stress $\sigma_{a p}$ estimated at four temperatures.

The dislocation (true) activation volume $V_{d}$ is obtained by the following equation:

$$
V_{d}=\left(\frac{\partial \Delta G}{\partial \tau^{*}}\right)_{\tau, s}=k T\left(\frac{\partial \ln \left(\dot{\gamma} / \dot{\gamma}_{0}\right)}{\partial \tau^{*}}\right)_{\tau, s},
$$

where the subscripts $T$ and s indicate that both the temperature and the dislocation microstructure (especially the mobile dislocation density) have to be constant during the test. Equation (14) is usually used for the activation volume estimation in tests with changes in strain rate (or machine speed). The $\dot{\gamma}_{0}$ term is essentially structure-dependent and can be expressed as

$$
\dot{\gamma}_{0}=\rho_{m} b \frac{A}{\ell_{c}} v_{D} \frac{b}{\ell_{c}} .
$$

where $A$ is the mean area swept by the dislocation segment per successful thermally activated event, $A=V \mid b, v_{D}$ is the Debye frequency and $\ell_{c}$ is the critical dislocation length for the thermally activated process to occur. In the stress relaxation experiments of polycrystals, it is only possible to record the variation of the applied stress, $\sigma_{a p}$, associated with the change in plastic strain rate (stress relaxation rate) and to determine an apparent activation volume, $V_{a p p}$, considering the mobile dislocation density and the internal stress as constant. The apparent activation volume, $V_{a p p}$, estimated in experiments with polycrystals is proportional to the dislocation activation volume as $V_{a p p}=(1 / \psi) V_{d}$. Apparent (experimental) activation volumes estimated for various temperatures are plotted against the thermal stress $\sigma^{*}$ in Fig. 4. All values appear to lie on one line, "master curve". Kocks et al. [10] suggested an empirical relation between the Gibbs enthalpy $\Delta G$ and the effective stress $\sigma^{*}$ in the following form:

$$
\Delta G=\Delta G_{0}\left[1-\left(\frac{\sigma^{*}}{\sigma_{0}^{*}}\right)^{p}\right]^{q},
$$

where $\Delta G_{0}$ and $\sigma_{0}^{*}$ are Gibbs enthalpy and the effective stress at 0 K. From Eqs. (5) and (16) it follows:

$$
\sigma^{*}=\sigma_{0}^{*}\left[1-\left(\frac{k T}{\Delta G_{0}} \ln \frac{\dot{\varepsilon}_{0}}{\dot{\varepsilon}}\right)^{1 / q}\right]^{1 / p},
$$

where $p$ and $q$ are phenomenological parameters reflecting the shape of a resistance profile. The possible ranges of values $p$ and $\mathrm{q}$ are limited by the conditions $0<p \leq 1$ and $1 \leq q \leq 2$. Ono $[11,12]$, suggested that Eq. (17) with $p=1 / 2, q=3 / 2$ describes a barrier shape profile that fits many predicted barrier shapes. Equation (17) can be rewritten

$$
\dot{\varepsilon}=\dot{\varepsilon}_{0} \exp \left[-\frac{\Delta G_{0}}{k T}\left(1-\left(\frac{\sigma^{*}}{\sigma_{0}^{*}}\right)^{p}\right)^{q}\right]
$$

and for the activation volume one obtains:

$$
V=k T \frac{\partial \ln \dot{\varepsilon} / \dot{\varepsilon}_{0}}{\partial \sigma^{*}}=\frac{\Delta G_{0} p q}{\sigma_{0}^{*}}\left[1-\left(\frac{\sigma^{*}}{\sigma_{0}^{*}}\right)^{p}\right]^{q-1}\left(\frac{\sigma^{*}}{\sigma_{0}^{*}}\right)^{p-1} .
$$

The values of the activation volume should lie at the curve given by Eq. (19). The fit of the experimental values (taken from Fig. 4) of Eq. (19) gives for the activation enthalpy $\Delta G_{0}=0.95 \pm$ $\pm 0.05 \mathrm{eV}$. (see Fig. 4). Similar values of the activation enthalpy were estimated for other magnesium alloys $[14,15]$. Using binominal expansion in Eq. (19), the activation volume should depends on the effective stress as $V_{a p p} \propto\left(\sigma^{*}\right)^{-n}$. Generally, the values of the power exponent found in the literature vary from -0.5 to -1 [10].

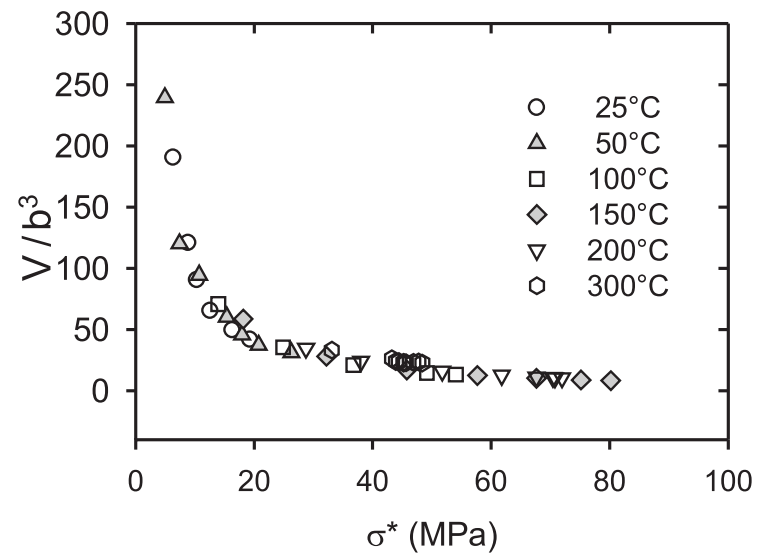

Fig. 4. Plot of the apparent activation volume (in $b^{3}$ ) against the thermal stress $\sigma^{*}$ estimated for various deformation temperatures

The activation enthalpy $\Delta H=\Delta G-T \Delta S$ ( $\Delta S$ is the entropy) is done by

$$
\Delta H=-T V_{d} \frac{\partial \tau^{*}}{\partial T} .
$$

For polycrystals, we can measure experimentally $\partial \sigma / \partial T$. Substituting from (14) into (20) it follows for the activation enthalpy 


$$
\Delta H=T \cdot V_{a p p}\left(\frac{\partial \sigma}{\partial \tau^{*}}\right)\left(\frac{\partial \sigma}{\partial T}\right)\left(\frac{\partial \tau^{*}}{\partial \sigma}\right)=-T V_{a p p}\left(\frac{\partial \sigma}{\partial T}\right) .
$$

The activation enthalpy calculated according to $(21)$ for $150{ }^{\circ} \mathrm{C}$ gives $(0.96 \pm 0.05) \mathrm{eV}$. Activation energies for high temperature creep of polycrystalline magnesium were measured originally by McG. Tegart [16] for various temperatures and stresses. He estimated that the activation energy is from room temperature up to $0.6 T_{m}\left(T_{m}\right.$ is the melting point in $\left.K\right)$ more less independent of temperature and slightly dependent on the applied stress. It was found for higher stresses to be $\sim 1 \mathrm{eV}$. Similar values were found for pure magnesium at $400 \mathrm{~K}$ by Milicka et al. [17] and Vagarali and Langdon [18] and for $\mathrm{Mg}-0.8 \% \mathrm{Al}$ [19].

The values of the activation volume and the activation enthalpy may help to identify thermally activated processes considering some of the common short-range barriers to dislocation motion [13]. The dislocation-dislocation interaction mechanism has an activation volume ranging from about $10^{2}-10^{4} b^{3}$, with the activation volume and enthalpy varying with strain. A rapid decrease in the internal stress with temperature (see Figs. 1d and 1e) indicates that softening is connected with dynamic recovery.

Magnesium alloys, with hcp structure, deform on many possible glide systems with dislocations of Burgers vector $\langle a\rangle=1 / 3$ [11 $\overline{2} 0]$ in basal, prismatic, and first-order pyramidal planes and with dislocations of Burgers vector $\langle c+a\rangle=1 / 3$ [11 $\overline{2} 3]$ in first- and second-order pyramidal planes. The main deformation mode in magnesium is basal slip of $\langle a\rangle$ dislocations. The secondary conservative slip may be realised by the $\langle a\rangle$ dislocations on prismatic and pyramidal planes of the first order. Couret and Caillard [20, 21] studied by TEM prismatic glide in magnesium in a wide temperature interval. They showed that screw dislocations with the Burgers vector $1 / 3[11 \overline{2} 0]$ are able to glide on prismatic planes and their mobility is much lower than the mobility of edge dislocations. The deformation is controlled by thermally activated glide of those screw dislocation segments. A single controlling mechanism was identified as the Friedel-Escaig cross slip mechanism. This mechanism assumes dissociated dislocations on compact planes, like (0001), that joint together along a critical length $L_{r}$ producing double kinks on non-compact planes. The activation volume is proportional to the critical length between two kinks. Amadieh et al. [22] found for the activation volume of the Friedel-Escaig mechanism a value of $70 b^{3}$. Prismatic slip was also observed by Koike and Ohyama [23] in deformed AZ61 sheets The activity of the $\langle a\rangle$ dislocations in prismatic planes is possible when the critical resolved shear stress ratio of prismatic to basal slip exhibits values $1.5-2$. It is realised at elevated temperatures and also at lower temperatures for a special texture of samples. The activation of the prismatic slip and subsequent annihilation of dislocation segments with opposite sign are probably the main reason for the observed internal stress decrease. The double cross slip may be a thermally activated process controlling the dislocation velocity. Beside this mechanism, the thermally activated glide of $\langle c+a\rangle$ dislocations should be taken into account. Mathis et al. [24] studied the evolution of non-basal dislocations as a function of temperature in magnesium by X-ray diffraction. They found a majority of $\langle a\rangle$ dislocations in the as-cast state. During plastic deformation in tension the $\langle a\rangle$-type dislocations remain dominant, however, the dislocation density increased by about a factor of three up to about $100{ }^{\circ} \mathrm{C}$. At higher temperatures the fraction of $\langle c+a\rangle$-type dislocations increased at the cost of $\langle a\rangle$-type dislocations and the increase of the dislocation density is strongly reduced. The internal stress acting on the dislocations is determined by the details of the internal structure at that moment and it is independent of the applied stress. The stress that changes when the applied stress is changed is only the effective stress. The internal stresses during plastic deformation of this alloy can be considered as the sum of stresses resulting from various dislocation arrangements and obstacles existing in the deformed material $[9,25]$. The solute atoms do not influence the moving dislocations at higher temperatures. However, at higher temperatures the solute atoms my diffuse to the staking fault and influence double cross slip from basal to non-basal planes.

\subsection{Strain ageing}

From the temperature dependence of the yield stress, introduced in Fig. 5, some local maximum between 50 and $100{ }^{\circ} \mathrm{C}$ is obvious. This maximum is a consequence of the dynamic strain ageing. It is also manifested at the stress strain curve by the so called post relaxation effect. When the internal stress as well as the dislocation density are constant than the deformation process continues at the same stress as at the beginning of the SR test. On the stress strain curve shown in Fig. 6 stress increase after SR test is obvious. The flow stress after the stress relaxation, $\sigma_{1}$, is higher than the flow stress at the beginning of the relaxation. The values of $\Delta \sigma=\sigma_{1}-\sigma_{0}$ are plotted against strain for two temperatures of 25 and $50{ }^{\circ} \mathrm{C}$ in Fig. 7. For other temperatures the post relaxation effect was not observed. From Fig. 7 it is to see that the strain dependence of $\Delta \sigma$ has some maximum at a certain strain.

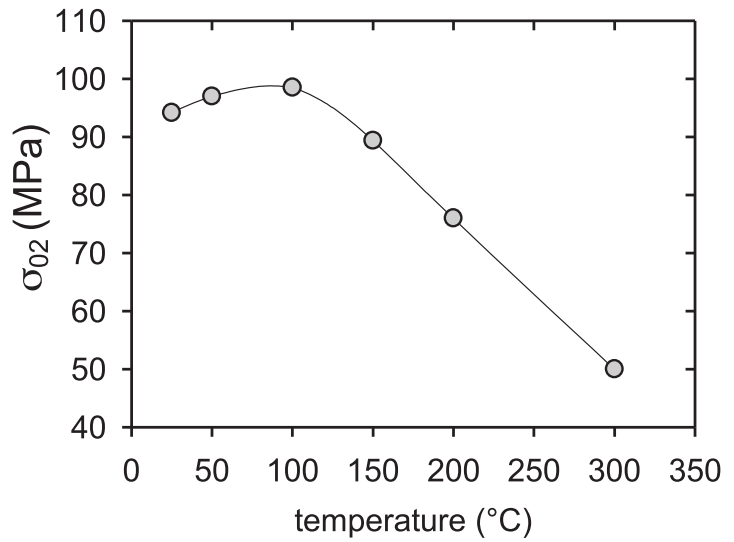

Fig. 5. Temperature dependence of the yield stress.

In an alloy the flow stress may be consider as a sum of two additive contributions:

$\sigma=\sigma_{f}+\sigma_{d}$ 


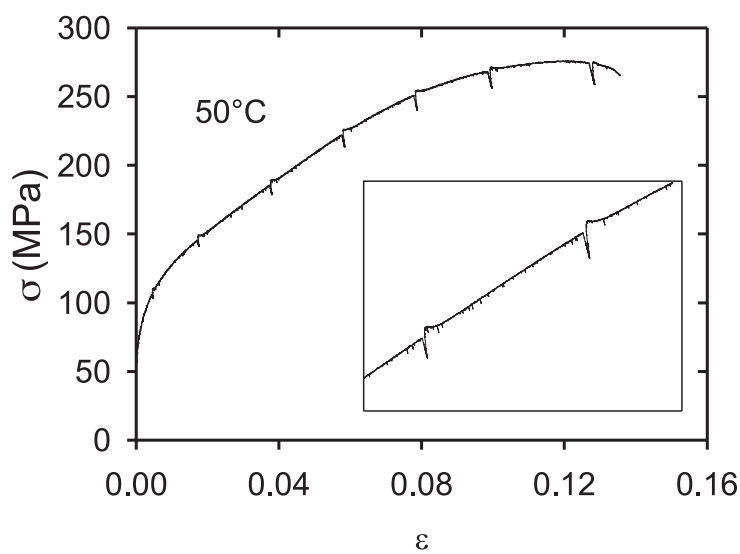

Fig. 6. The stress-strain curve obtained at $50{ }^{\circ} \mathrm{C}$. An increase of the stress after the stress relaxation test is from the insert well visible.

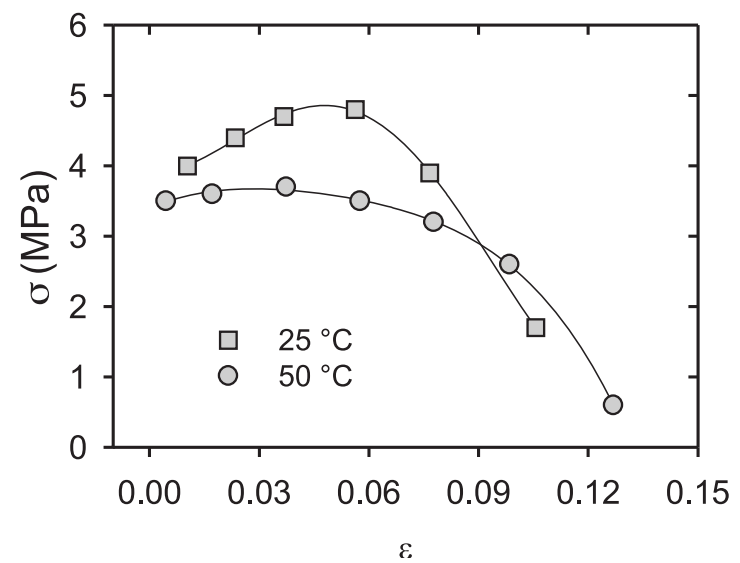

Fig. 7. The stress increase $\Delta \sigma$ depending on the strain estimated for 25 and $50{ }^{\circ} \mathrm{C}$.

with $\sigma_{f}$ relating to a friction imposed by the solutes-dislocation interaction $\sigma_{d}$ relating to the dislocation-dislocation interaction. Hong $[26,27]$ suggested that the stress $\sigma_{f}$ could be described by the following equation:

$$
\sigma_{f}=\alpha_{1} G B \delta c \exp \left(-\frac{\left(T-T_{0}\right)^{2}}{B}\right)
$$

where $\alpha_{1}$ is a constant, $\delta$ is the atomic size misfit parameter, $c$ is the solute concentration and $B$ is the width of the distribution about the temperature $T_{0}$ where the maximum of solute-dislocation interaction force occurs. The critical dislocation velocity $V_{c}$ at which the maximum force occurs can be expressed as:

$$
V_{c}=\alpha_{2} \frac{k T_{0} D_{0}}{G b \delta \Omega} \exp \left(-\frac{Q_{D}}{k T}\right)
$$

where $\alpha_{2}$ is a constant and $D_{0}$ is the diffusion constant for solute atoms, $\Omega$ is the atomic volume and $Q_{D}$ is the activation energy for diffusion of solute atoms in magnesium matrix. The critical strain rate at which the maximum interaction stress occurs can be predicted using the following equation:

$$
\dot{\varepsilon}_{c}=\rho_{m} b \frac{\pi(1-v) k T_{0} D_{0}}{(1+v) G b \delta \Omega} \exp \left(-\frac{Q_{D}}{k T}\right),
$$

where $v$ is the Poisson ratio, $\rho_{m}$ is the mobile dislocation density. From Eq. (23) it can be seen that the friction force due to solute atoms interaction with moving dislocations exists only in a certain temperature interval depending on solute atoms type. This friction force (stress) is added to the temperature dependence of the yield stress resulting to a local maximum in the temperature dependence of the yield stress. Such local maximum in the temperature dependence is demonstrated in Fig. 5. Similar local maximum was observed in the case of ZE41 alloy [28], AZ91 alloy [29] of binary alloy $\mathrm{Mg}-0.7 \mathrm{Nd}[30]$.

According to Malygin [31] and Rubiolo and Bozzano [32] solute atoms diffuse to dislocations arrested at local obstacles for waiting time $t_{w}$. The concentration of solute atoms at dislocation lines as a function of the waiting time $c\left(t_{w}\right)$ is done by the function

$$
c=c_{m}\left[1-\exp \left(-\frac{c_{0}}{c_{m}}\left(\alpha_{n} D t_{w} / b^{2}\right)\right)^{2 /(n+2)}\right],
$$

where

$$
\alpha_{n}=n(n+2) \pi^{(n+2) / 2}\left(W_{b} / k T\right) .
$$

$c_{0}$ is the average concentration of impurities in the crystal and $c_{m}$ is the limiting value of impurities on the dislocations. $W_{B}$ is the binding energy of solute atoms to dislocations. The value of $n$ depends on the details of interaction between solute atom and dislocations. The exponent in eq. (26) $r=2 /(n+2)$ is typically $2 / 3$ and $1 / 3$ for bulk and pipe diffusion, respectively. [33].

The stress increment $\Delta \sigma$ after SR due to solute atoms segregation may be also expressed for longer time by the following equation

$$
\Delta \sigma(t, \varepsilon, T)=\Delta \sigma_{m}(\varepsilon, T)\left\{1-\exp \left[-\left(t / t_{c}\right)^{p}\right]\right\},
$$

where $\Delta \sigma_{m}(\varepsilon, T)$ is the stress increment for $t \rightarrow \infty$ and it depends on the binding energy between solute atoms and dislocations. (It increases with increasing solute atom concentration and with decreasing temperature.) $t_{c}$ is a characteristic time which depends on the strain as $t_{c} \sim \varepsilon^{-k}$. [34]. Solute atoms locking dislocations cause the stress increase after stress relaxation, which depends on strain and on temperature. An increase in the flow stress is needed to move the dislocations after stress relaxation. It is reasonably to assume that $\Delta \sigma$ is proportional to the number of impurities on dislocation lines.

\section{Conclusions}

The stress relaxation tests were performed during compression experiments at various temperatures with the aim to reveal the main features of the plastic deformation mechanisms. The complex analysis of the stress relaxation curves showed: 
- the internal stress decreases with increasing deformation temperature;

- the values of the apparent activation volumes are in the order of tens to hundreds $b^{3}$;

- the activation volume depends on the thermal stress so that all values lie at the master curve $V_{a p p} \propto\left(\sigma^{*}\right)^{n}$;

- the estimated activation energy indicates that the main thermally activated process is very probably the glide of dislocations in the non-compact planes,

- mobile solute atoms are the reason for the dynamic strain ageing manifested by the post relaxation effect and the local maximum in the temperature dependence of the yield stress.
Acknowledgement. The authors dedicate this paper to Prof. Ing. Otakar Bokuvka, $\mathrm{PhD}$ on the occasion his $65^{\text {th }}$ birthday. This work is a part of the research plan 1M2560471601 "Eco-center for Applied Research of Non-ferrous Metals" that is financed by the Ministry of Education, Youth and Sports of the Czech Republic. This work was also supported by the Grant Agency of the Academy of Sciences of the Czech Republic under Grant IAA201120902.

\section{References}

[1] POLMEAR, I. J.: Magnesium alloys, Light Alloys (Fourth Edition), 2005, 237.

[2] AVEDESIAN, M. M., BAKER, H. (Eds.): Magnesium and Magnesium Alloys, ASM Specialty Handbook, ASM International, Materials Park, OH, 1999.

[3] ROBERTS, C. R.: Magnesium and Its Alloys, John Wiley \& Sons 1960.

[4] MORDIKE, B. L.: J. JILM 51, 2001, 2.

[5] LI, J. C. M.: Canad. J. Appl. Phys. 45, 1967493.

[6] DE BATIST, R., CALLENS, A.: Phys. Stat. Sol. (a) 21, (1974) 591-595.

[7] DOTSENKO, V.I.: Phys. Stat. Sol. (b) 93, 1979, 11.

[8] FELTHAM, P.: Phys. Stat. Sol. 3, 1943, 1340.

[9] MILICKA, K., TROJANOVA, Z., LUKAC, P.: Mater. Sci. Eng. A 462. 2007. 215.

[10] KOCKS, U. F., ARGON, A. S., ALBY, M. F.: Progr. Mater. Sci. 19. 1975. 1

[11] ONO, K.: J. Appl. Phys. 39. 1968. 1803.

[12] KAPOOR, R., WADEKAR, S. L., CHAKRAVARTTY, J. K.: Mater. Sci. Engn. A 328, 2002, 324.

[13] EVANS, A.G., RAWLINGS, R. D.: Phys. Stat. Sol. 34, 1969, 9.

[14] TROJANOVA, Z., LUKAC. P., KAINER, K. U.: Advanced Engn. Mater. 9, 2007, 370.

[15] TROJANOVA, Z., LUKAC, P.: Inter. J. of Mater. Research 100, 2009, 270.

[16] MCG. TEGART, W. J.: Acta Metall. 9, 1961, 614.

[17] MILICKA, K., CADEK, J., RYS, P.: Acta Metall. 18, 1970, 1071.

[18] VAGARALI, S. S., LANGDON, T.G.: Acta Metall. 29, 1982, 1969-1982.

[19] VAGARALI, S. S., LANGDON, T.G.: Acta Metall. 30, 1982, 1157.

[20] COURET, A., CAILLARD, D.: Acta metall. 33 (1985) 1447.

[21] COURET, A., CAILLARD, D.: Acta metall. 33 (1985) 1455.

[22] AMADIEH, A., MITCHELL, J., DORN, J. E.: Trans. AIME 233, 1965, 1130.

[23] KOIKE, J., OHYAMA, R.: Acta Mater. 53, 2005, 1963.

[24] MATHIS, K., NYILAS, K., AXT, A., DRAGOMIR-CERNATESCU, I., UNGAR, T., LUKAC, P.: Acta Mater. 52, $2004,2889$.

[25] TROJANOVA, Z., LUKAC, P., MILICKA, K., SZARAZ, Z.: Mater. Sci. Eng. A 387-389, 2004, 80.

[26] HONG, S. I.: Mater. Sci. Eng. A110, 1989, 125.

[27] HONG, S. I.: Mater. Sci. Eng. 91, 1987, 137.

[28] TROJANOVA, Z., LUKAC, P.: Kovove Mater. 43, 2005, 73.

[29] TROJANOVA, Z., LUKAC, P., GABOR, P.: Kovove Mater. 39, 2001, 368.

[30] TROJANOVA. Z., LUKAC. P.: In: Magnesium Alloys and their Applications 8, Ed. K.U. Kainer, DGM 2009, Willey VCH Weinheim, 785-802.

[31] MALYGIN, G. A.: Phys. Stat. Sol. (a) 1982, 72, 493.

[32] RUBIOLO, G. H., BOZZANO, P. B.: Mater. Trans. JIM 36. 1995, 1124.

[33] BALIK, J., LUKAC, P.: Kovove Mater. 36, 1998, 3.

[16] LUBENETS, S. V., STARTSEV, V. I., FOMENKO, L.S.: Czech. J. Phys. B 36, 1986, 493. 